\title{
Las comunidades académicas
} y las redes de información en Ciencias Sociales en América Latina: la cooperación como estrategia de sobrevivencia y puente para el conocimiento en tiempos difíciles *

\author{
Martha Sabelli **
}

Artículo recibido:

7 de mayo de 2012.

Artículo aceptado:

27 de junio de 2012.

\section{RESUMEN}

Los centros privados de investigación durante el período autoritario en América Latina constituyeron ONG donde destacados sociólogos, demógrafos, politólogos y otros especialistas de ciencias sociales (que habían tenido un lugar destacado en las universidades públicas hasta su intervención por las dictaduras militares durante los años 70 y 80) generaron conocimiento y líneas de investigación sobre las sociedades de la época. Estos reductos de la "intelligentsia" latinoamericana que pudieron permanecer en la región trabajaron estrechamente con los centros de

Presentada en la Reunión Satélite de Ciencias Sociales: Bibliotecas de Ciencias Sociales: un puente hacia el conocimiento necesario para el Desarrollo Sostenible. Sección de Bibliotecas de Ciencias Sociales de la IFLA.Biblioteca de Cuba José Martí, 8-10 de agosto de 2011.

** Universidad de la República, Uruguay. marthasabelli@gmail.com

INVESTIGACIÓN BIBLIOTECOLÓGICA, Vol. 26, Núm. 57, mayo/agosto, 2012, México, ISSN: 0187-358X. pp. 233-247 
documentación y bibliotecas. Esto creó un diálogo fecundo y cotidiano entre los profesionales de la información y los académicos, o sea, en y con dominios específicos y comunidades de práctica. El análisis de las redes y recursos de información de aquel período promovidas por CLACSO, DOCPAL de CELADE, CLADES de CEPAL, a la luz de las teorías del análisis del dominio y de la información de la vida cotidiana, ofrece una rica experiencia y aprendizaje a las bibliotecas y servicios de información en ciencias sociales actuales. Los comportamientos informativos de los científicos sociales interrelacionados e insertos en el contexto de los servicios de información y documentación funcionando en redes regionales forman parte de un modelo a difundir y discutir.

Palabras claves: Redes de información en ciencias sociales; bibliotecas y centros de documentación en ciencias sociales; América Latina 1975-1985; DOCPAL-CELADE; CLADES-CEPAL; CLACSO

ABSTRACT

Academic communities and information networks in Social Sciences in Latin America: cooperation as a survival strategy and bridge for knowledge trough difficult times

Martha Sabelli

Private research centers during the authoritarian period in Latin America were constituted by NGO's where prominent sociologists, demographers, political scientists and other specialists in social sciences (who had reached prominent positions in public universities until the military dictatorships intervention during the 70s and 80s), generated knowledge and lines of research with reference to the societies of the time. These strongholds of Latin American "intelligentsia" - which could remain in the region- worked closely with libraries and documentation centers. As a result, this created a fruitful dialogue between information professionals and academics, i.e., in and with specific domains and communities of practice. The analysis of networks and information resources of the period promoted by CLACSO, DOCPAL from CELADE and CLADES from CEPAL, through theories of domain analysis and daily 
life information, offers vast experience and training to libraries and information services in social sciences nowadays. Information behaviors of social scientists which interrelated and inserted in the context of information and documentation services operating in regional networks, became part of a model to disseminate and discuss.

Keywords: Information networks in Social Sciences; Social Sciences Libraries and Documentation Centers; Latin America 1975-1985; DOCPALCELADE; CLADES-CEPAL; CLACSO

\section{LOS CENTROS DE INVESTIGACIÓN EN CIENCIAS SOCIALES EN EL PERÍODO AUTORITARIO Y TRANSICIÓN DEMOCRÁTICA Y LAS UNIDADES DE INFORMACIÓN}

T os centros de investigación durante el período autoritario en América otros especialistas de ciencias sociales, que habían tenido un lugar destacado en las universidades públicas hasta su intervención por los gobiernos (años 70 y 80), generaron conocimiento y líneas de investigación sobre las sociedades de la época. Estos reductos de la "intelligentsia” latinoamericana que pudieron permanecer en la región - trabajaron estrechamente con los centros de documentación y bibliotecas de sus instituciones. Esto creó un diálogo fecundo y cotidiano entre los profesionales de la información y los académicos, o sea, en la comunidad de práctica.

Abordaremos el rol de las redes y recursos de información de aquel período (CLACSO, CELADE/DOCPAL, CEPAL/CLADES ${ }^{1}$ ) a la luz de las teorías del análisis del dominio y de la información en la vida cotidiana. Ello ofrece un rico campo de experiencias y aprendizajes sobre los comportamientos informativos de investigadores interrelacionados e insertos en el contexto de los servicios de información y documentación funcionando en redes regionales.

Pensamos que la situación vivida por las ciencias bibliotecológicas y de información en el desarrollo de las ciencias sociales en América Latina en este

1 CLACSO-Centro Latinoamericano de Ciencias Sociales; CELADE-Centro Latinoamericano de Demografía; DOCPAL-Sistema de Documentación sobre Población en América Latina; CEPALComisión Económica para América Latina y el Caribe /; CLADES-Centro Latinoamericano de Documentación Económica y Social. 
lapso, con la presencia de numerosos centros académicos de investigación autónomos, de dimensiones pequeñas pero con una importante creación de conocimiento, no está debidamente reconocida y recordada por la historia — aún por escribirse- del desarrollo de la disciplina y del quehacer profesional en esta área.

Estos institutos desempeñaron un muy importante papel durante los gobiernos autoritarios del período, ya que sus miembros, valiosos académicos y expertos, fueron expulsados de las universidades públicas y en ellos pudieron seguir investigando y entablando relación con sus pares del exterior. Se trabajaba con base en proyectos concursados, seleccionados, apoyados y evaluados rigurosamente desde Europa, Estados Unidos y Canadá por fundaciones y organizaciones internacionales.

Las unidades de información (bibliotecas y/o centros de documentación e información) de dichos institutos trabajaron en forma interdisciplinaria con los investigadores y participaron en redes de información, promovidas desde DOCPAL, CLADES y CLACSO, y un sector de ellas fueron apoyadas al igual que los investigadores- por distintos organismos y fundaciones. Lo importante a destacar en esta Reunión, que apunta al presente y futuro próximo, es que en aquellos tiempos y contextos existió una integración entre la teoría y la práctica y una profusa interdisciplinariedad. Abordamos esta temática en el Conferencia CoLIS 7 (The Seventh International Conference on Conceptions of Library and Information Science: "Integration in the Information Sciences: Unity in Diversity), donde afirmamos que

[...] seguramente la situación política, los ámbitos reducidos y fermentales de convivencia entre investigadores y profesionales de la información, en las bibliotecas, pero también en los corredores, en las oficinas, a la hora de los almuerzos compartidos, promovieron una actitud y aptitudes para la investigación en información y documentación (Sabelli, 2010).

Los centros de investigación en ciencias sociales durante el período autoritario han sido estudiados por distintos autores, quienes los han denominado centros de investigación o centros académicos. Si bien varían en su caracterización: "independientes" (Brunner y Barrios, 1987), "autónomos" (Prates, 1987) o "privados" (Barreiro Díaz, 2000) observamos que coinciden en su identificación. Otros optan por llamarlos sin adjetivos "centros de investigación" (Paris de Oddone y Alfaro, 1987). Cabe señalar que esta bibliografía refiere al caso uruguayo, excepto el libro de Brunner y Barrios que abarca a los países del Cono Sur: Argentina, Brasil, Chile y Uruguay y realiza un estudio comparativo entre ellos, correspondiente al período autoritario y al lapso considerado de "redemocratización". 
Nos referiremos al caso uruguayo, pues la perspectiva de este artículo se basa en las experiencias y reflexiones desde un centro de investigación en Uruguay: CIESU. El contexto corresponde al gobierno autoritario (1973-1984) y los primeros tiempos de la restauración democrática (1985 y los inicios de los años 90), que podemos considerarlos también difíciles para la Universidad de la República, que concentraba antes de 1973 casi la totalidad de la generación del conocimiento y la enseñanza superior, y debió realizar un gran esfuerzo en restablecer a sus docentes, investigadores, nuevos planes de estudio, y en definitiva, su reinserción en la sociedad uruguaya. En suma, dar respuesta al desmantelamiento de la Universidad que se había realizado durante el período de represión cultural. De allí que tomemos un lapso más amplio, pues la reestructura de la investigación fue un proceso de algunos años.

CIESU fue uno de los cinco centros miembros de CLACSO del período señalado (CIEDUR, CIEP, CIESU, CINVE, CLAEH) y existe consenso en considerar el importante aporte que hicieron para repensar al Uruguay en crisis.

\section{LOS CENTROS DE DOCUMENTACIÓN REGIONALES Y LAS REDES DE UNIDADES DE INFORMACIÓN}

De este período desde fines de los años 70 hasta inicios de los 90, y en estas áreas de investigación y ejercicio profesional, debemos rescatar y valorizar el rol desempeñado por el área de Ciencias de la Información desde 1970 a 1995 del Centro Internacional de Investigaciones para el Desarrollo CIID/ IDRC en América Latina, apoyando programas y proyectos de información y documentación dirigidos a promover el desarrollo social. Asimismo DOCPAL, CLADES y CLACSO constituyeron centros generadores de conocimiento, teoría y difusión de tecnología para el procesamiento de información y de intercambio académico en eventos regionales convocados por estos centros regionales. Allí se reunían los profesionales de la información con investigadores y planificadores. La Serie Información y Desarrollo de CLADES, publicada hacia el final de este período, es la expresión más clara del afán de unir la teoría a la práctica profesional en un contexto de las disciplinas de la información.

No podemos dejar de recordar aquí los aportes desde Chile de Betty Johnson (DOCPAL), de Claudionor Evangelista y Julio Cubillo (CLADES) y desde Argentina de Dominique Babini (CLACSO) así como los equipos de dichas instituciones, y desde el ámbito de la informática en este período a Abel Packer (DOCPAL y CLADES).

En este intercambio en reuniones y a la distancia (nos comunicábamos por correo electrónico por la red Chasque antes de la existencia de Internet) no 
había fragmentaciones entre las distintas áreas y disciplinas de información, ni entre sus distintos profesionales. Creemos que hay mucho que aprender de aquel período, caracterizado por la automatización, la creación de las bases de datos, los catálogos automatizados, el desarrollo y empleo de tesauros y, muy especialmente, por participar en forma conjunta de recursos gratuitos de alta calidad y una estrecha cooperación e intercambio en redes. No existía o era muy débil la fragmentación de entre la teoría y la práctica a nivel de la disciplina y el quehacer profesional. Se compartía la teoría y práctica del análisis de información, la gestión de los servicios, la incorporación de las tecnologías (software MicroISIS), los formatos, etcétera.

A continuación presentaremos brevemente estos espacios de cooperación creados y desarrollados en la región, con el fin de compartir una reflexión sobre la cooperación como estrategia de sobrevivencia y puente para el conocimiento en estos tiempos difíciles.

\subsection{DOCPAL y la Red IPALCA: un modelo de transferencia de metodologías y conocimientos a los centros participantes}

El Centro Latinoamericano de Demografía CELADE creó el Sistema de Documentación sobre Población en América Latina y el Caribe DOCPAL en 1976, con el aporte financiero del CIID / IDRC de Canadá. Constituyó uno de los primeros servicios de los países en desarrollo que utilizaron la computación para procesar y recuperar los documentos. El sistema utilizado fue el ISIS, diseñado por IDRC, con formatos de entrada y salida de datos unificados pocos años después con CEPAL / CLADES y la Biblioteca de CEPAL en un sistema de gestión de información bibliográfica basado en normas internacionales.

A fines de los años 70 e inicios de los 80, DOCPAL consolidó un Servicio de Documentación modelo a nivel mundial, tanto por la excelente calidad de los instrumentos elaborados ("Manuales de Documentación sobre Población" publicados en la Serie B de CELADE), como por su visión sobre la futura automatización de los catálogos de las unidades de información (bibliotecas, centros de documentación y servicios de información) de la región. Nos referimos al diseño de las TRB (Tarjetas de Registro Bibliográfico) que permitían a las unidades ingresar la catalogación, indización y resúmenes en campos de registro codificados con vistas a conformar catálogos automatizados con relativa facilidad. Las TRB se podían utilizar con el sistema unitérmino, que era brindado a través de fichas preparadas para tal fin. Cabe destacar el importante rol del Tesauro de la Red de Información sobre Población de las Naciones Unidas en la normalización del vocabulario y, por tanto, en la coordinación de la indización y recuperación de información desarrolladas por las unidades especializadas en población a nivel regional. 
En suma, la metodología de DOCPAL permitió trabajar en forma manual pero logrando un muy rápido y fácil ingreso de los datos a un sistema computarizado. Pensemos que estábamos aún con la presencia de computadores de gran porte, solamente las poseían grandes organizaciones e instituciones públicas y privadas. Cuando llegaron los microcomputadores en la segunda mitad de los años 80 al mercado de la región, las unidades de información los incorporaron gradualmente. Aquéllas que poseían esta metodología para el tratamiento de la documentación pudieron en un corto lapso construir sus bases de datos bibliográficas al utilizar el software de información bibliográfica MicroISIS.

En nuestro caso, desde el Centro de Documentación del Centro de Informaciones y Estudios del Uruguay CIESU, después de una pasantía en julio de 1980 en DOCPAL, iniciamos la gestión documental con dicha metodología, lo que permitió en 1987 automatizar el catálogo de TRB y crear — a través de un proyecto (1988-1990) apoyado por el CIID— un Servicio de Información en Urbanización basado en las técnicas y herramientas transferidas por DOCPAL.

Los impactos más importantes de DOCPAL y de su equipo de profesionales, liderado por Arthur Conning y Betty Johnson, radicaron por un lado en la formación en el servicio de bibliotecarios y/o documentalistas latinoamericanos a través de pasantías, y por otro, en la promoción de la coordinación, el intercambio y, en definitiva, el acercamiento entre los centros participantes, a través de Seminarios regionales y el nacimiento de la Red IPALCA (Red de Información sobre Población en América Latina y el Caribe) en 1987. ${ }^{2}$

Vemos pues que la relación entre la autosuficiencia y la interdependencia de las instituciones para responder a las necesidades de información estuvo en la base de la construcción de los sistemas regionales e internacionales emergentes en este período.

Una característica de los Seminarios fue la participación de científicos sociales, los planificadores y otros usuarios conjuntamente con los documentalistas "con el objeto de estimular el diálogo entre todos los involucrados en los procesos de generación y uso de información” (IPALCA, 1987). De esta manera, la propuesta de estrategias y proyectos a emprender emergían de un "dominio" específico centrado en la temática población y reunía a actores que se desempeñaban como generadores, mediadores y usuarios de información documental. El contexto de crisis de la llamada "década perdida"

2 Recordamos especialmente: Seminario Información sobre Población para el Desarrollo, Estrategias y Propuestas para satisfacer los requerimientos de América y el Caribe durante la próxima década (1987-1996), CELADE/PROLAP, 28-31 de julio 1987 y el Seminario Red IPALCA: aprovechando la nueva tecnología de información y comunicación, Santiago de Chile, 16-17 de noviembre de 1993 
incentivaba a reducir la atomización de los sistemas de información y maximizar su interdependencia con todas las instituciones que trabajaban en el mismo campo, y conllevaba el compartir recursos de información.

Es interesante observar en las primeras etapas del período democrático, y tras el consiguiente debilitamiento de los apoyos económicos a las redes y sistemas regionales desde el exterior de la región, una preocupación por un mejor aprovechamiento de las redes. Un mayor acceso y uso de los recursos centra las preocupaciones y orienta las recomendaciones. Reconocer a la comunidad de usuarios, sus necesidades y capacitación en el uso de las fuentes de información dentro de las universidades y otras instituciones aparece con frecuencia como un tema central a tomar en cuenta para una mejor gestión de los recursos de información. El documento base del Seminario del año 1993 (IPALCA, 1993) expresa estas inquietudes y, de alguna forma, cierra una etapa que debe ser recordada y valorizada en un contexto determinado por una multiplicidad de procesos inherentes a la evolución de la disciplina y el quehacer profesional en el proceso de informatización de la sociedad y a las coyunturas políticas y sociales de los países de la región.

\subsection{CLADES: los instrumentos tecnológicos y los aportes a la teoría de la gestión de la información en el contexto de "los cambios globales"}

El legado de CLADES puede identificarse en varias áreas, pero desde nuestra mirada diferenciamos: por un lado, los instrumentos creados para el tratamiento de la información ("Sistema de Información Bibliográfica de la CEPAL") y, por otro, los aportes a la reflexión y debate de la gestión de la información en el entorno de los cambios provocados por las TIC en los años 90.

El Sistema de Información Bibliográfica de CLADES, que tiene como antecedentes los procedimientos diseñados en las primeras etapas de la conformación de DOCPAL, significó para cientos de organizaciones no gubernamentales de investigación en ciencias sociales y/o promoción social de América Latina y el Caribe una ayuda difícil de evaluar. Sin el apoyo brindado a través de la transferencia gratuita y solidaria de "paquetes" tecnológicos de alta calidad, sencillos y amigables, la creación de bases bibliográficas no hubiera sido posible de incorporar por las unidades de información caracterizadas por sus escasos recursos. Sin duda, fue un ejemplo mundial de una manera cooperativa de enfocar los problemas en países en situaciones difíciles, no solamente por su condición de pobreza y crisis económica sino por los 
regímenes políticos de la época que no desarrollaban ni apoyaban políticas de información. ${ }^{3}$

Este compromiso con la promoción de una forma del "hacer" en documentación se efectivizó con un conjunto de manuales de procedimientos de información bibliográfica y cursos de formación, ${ }^{4}$ cuyos diseños y contenidos siguen siendo destacados por su calidad y claridad. Vemos, por tanto, cómo el rigor profesional acompañó la adopción de formas facilitadoras para una amplia gama de posibles destinatarios.

Aún hoy se sigue hablando y también usando, a lo largo y ancho de la región, los llamados "Formatos CEPAL" al considerar las hojas de entrada de registros y formatos bibliográficos de salida. Creemos que hay que dimensionar y valorizar el impacto que produjo a mediados de los años 80 e inicios de los $90 \mathrm{y}$, especialmente, considerar las posibilidades brindadas para el intercambio de información a nivel nacional y regional.

En este breve recorrido, debemos considerar la preocupación por la normalización del vocabulario en la indización y recuperación de información. Sin duda, el uso de Macrothesauro de OCDE al igual que el Tesauro de POPIN por DOCPAL fueron pasos importantes en los avances de la documentación latinoamericana en ciencias sociales.

Sin embargo, queremos poner énfasis en la contribución del equipo de CLADES, especialmente recordamos a Claudionor Evangelista y Martha Beya, quienes promovían el acercamiento e interrelación entre los encargados de las bibliotecas y centros de documentación de la región, donde eran — como suele pasar - tan importantes las horas del café y reuniones al final de la jornada como valiosos los contenidos de los cursos de capacitación. Esto se tradujo en importantes lazos personales e institucionales que fortalecieron las redes en desarrollo.

El segundo "legado" al que hacíamos referencia, los aportes a la reflexión y debate de la gestión de la información, tienen como principal responsable a un líder regional de la ciencia de la información en América Latina y el Caribe en los años 90, Julio Cubillo. Aunque podemos ubicar estas contribuciones hacia el final del período abordado en esta presentación, ellas fueron por diversas razones altamente significativas para las unidades de información en el período de restablecimiento democrático.

3 Estas afirmaciones deben ser consideradas con limitaciones debido a la escasa investigación sobre las políticas de información y el desarrollo bibliotecológico en el período; podemos arriesgar que no podemos incluir con la misma certeza a Brasil.

4 En el este período recordamos: Curso Regional para operadores de MICROISIS, organizado por CEPAL/CLADES y el CLAD, Santiago, Chile, 9-13 de mayo de 1988 y Cursillo de Adiestramiento en el Programa MICROISIS, CELADE, Santiago, Chile, 3-4 de agosto de 1987. 
La construcción de las redes y la consolidación de las unidades de información se podían realizar según modelos encapsulados y reducidos a la aplicación de técnicas y herramientas documentales, o darle un sentido basado en la reflexión sobre nuevas formas de gestión de la información y nuevos enfoques del gestor de información. En ese sentido Cubillo promovió el diálogo y el debate entre actores muy diversos: bibliotecarios, documentalistas, generadores de información y tomadores de decisión. ${ }^{5}$ Sus reflexiones y las emergentes de las reuniones se plasmaron en una Serie de publicaciones de CEPAL: "Información y Desarrollo" (NU. CEPAL (1990-1997)), ocho números editados entre 1990 y 1997. Sus temas son ilustrativos acerca de los ejes temáticos: los primeros números dirigidos a la planificación estratégica de los sistemas de información documentales y "ciclos de entrenamiento", y los últimos sobre los sistemas de información y los cambios globales y los nuevos gestores de información. Este tema que es representativo de las interrogantes y desafíos que cierran nuestro período ya está latente en los primeros años de los 90 .

Por todas estas razones, CLADES al igual que DOCPAL tuvo una doble virtud, adoptó perspectivas técnicas y a la vez perspectivas teóricas sobre la ciencia de la información en un contexto - como bien marcaban en sus discursos- de "cambios globales" que hacía preciso reposicionarse en los nuevos escenarios.

\subsection{CLACSO: promoviendo redes y sistemas de información desde los años 80}

CLACSO es desde su fundación en 1967 la mayor red académica de ciencias sociales en América Latina y el Caribe. Es una institución internacional nogubernamental que nuclea en la actualidad 293 centros de investigación y más de 500 programas de posgrado en Ciencias Sociales (maestrías y doctorados), radicados en 25 países de América Latina y el Caribe, en Estados Unidos y en Europa.

Los objetivos del Consejo son la promoción y el desarrollo de la investigación y la enseñanza de las Ciencias Sociales, así como el fortalecimiento del intercambio y la cooperación entre instituciones e investigadores de dentro y fuera de la región. Del mismo modo, promueve la activa diseminación del conocimiento producido por los científicos sociales en los movimientos sociales, las organizaciones populares y las entidades de la sociedad civil. A través de estas actividades, CLACSO contribuye

5 Un interesante debate se dio en la Reunión Regional sobre Gestión de Información, realizada 8-10 de junio de 1994. 
a repensar, desde una perspectiva crítica y plural, la problemática integral de las sociedades latinoamericanas y caribeñas. ${ }^{6}$

Todos somos conscientes del relevante rol que ha cumplido su Red de Información y cumple desde los años 2000 la Biblioteca Virtual de Ciencias Sociales de América Latina y el Caribe de la Red de Centros miembros de CLAC$S O$, ofreciendo servicios y recursos de información en forma gratuita desde el Área de Información y Documentación en Buenos Aires. Seguramente en esta reunión compartiremos las experiencias y logros de este trabajo desarrollado en forma cooperativa, descentralizada y en plataformas de acceso abierto. Es un ejemplo mundial en el ámbito académico de creación de bibliotecas virtuales de libre acceso basadas en la cooperación y trabajo participativo.

La preocupación por políticas de información a nivel regional y mundial tiene importantes antecedentes en el período que trata este trabajo. El Secretario Ejecutivo Francisco Delich (1976-1983) solicitó al Área de Documentación del CIESU un anteproyecto de Sistema de Información (Sabelli y Chiacchio, 1980), mostrando un gran interés en construir una red de información, así como por nuestra asistencia representando a CLACSO en el Curso Regional Latinoamericano sobre Recopilación, Almacenamiento, Difusión y Datos Socio-Económicos, realizado en Quito, Ecuador, 24 de noviembre - 4 de diciembre de 1980, organizado en CIESPAL por la Facultad Latinoamericana de Ciencias Sociales- FLACSO, donde comenzamos a compartir experiencias entre profesionales bibliotecarios y/o documentalistas e investigadores de la región.

En 1984 Dominique Babini inicia la coordinación del Área Información de CLACSO. Habíamos destacado al inicio de este ítem el relevante papel que en el período de este trabajo desempeñó —y sigue desempeñando con la capacidad y liderazgo que la caracteriza - como creadora e impulsora de proyectos cooperativos en el ámbito de las unidades de información en ciencias sociales del período. Su visión de las políticas de información para la región y su acertada comprensión de las etapas a cumplir hicieron posible la concreción de redes regionales e internacionales hasta la actualidad.

Babini coordinó desde 1992 a 1998 la International Development Information Network (IDIN) desarrollada en cinco regiones del mundo: Asia y el Pacífico (ADIPA-Association of Development Research and Training Institutes of Asia and the Pacific), Países Árabes (AICARDES-Association of Arab Institutes and Centres for Research in Economic and Social Development), América Latina (CLACSO-Consejo Latinoamericano de Ciencias Sociales), 
África (CODESRIA-Council for Development of Economic and Social Research in Africa) y Europa (EADI-European Association of Development Research and Training Institutes).

En ese marco difundieron la Base de datos de investigaciones en curso, la Base de datos de instituciones, la Base de datos de especialistas y se publicaron Directorios de Investigaciones y de Instituciones. Al igual que DOCPAL y CLADES, compartieron con las unidades de información de los centros miembros los formatos de las bases de datos en MicroISIS, diseñados por la Red IDIN y CLACSO.

Asimismo las reuniones regionales organizadas por la Red de Información de $\mathrm{CLACSO}^{7}$ fomentaron el intercambio entre las unidades de información en el contexto del desarrollo de la investigación de los centros miembros.

En suma, es válido y relevante hacer partícipes a los jóvenes colegas del rol del Área y Red de Información de CLACSO en aquellos tiempos, como coherente y consistente antecedente de la actual Biblioteca Virtual que tanto nos enorgullece en América Latina y el Caribe. No está de más acentuar la importancia del papel desempeñado en períodos de debilidades — reinicios e inicios- de las unidades de información en ciencias sociales de la región.

\section{UNA MIRADA DESDE UNO DE LOS CENTROS DE INVESTIGACIÓN: CIESU}

El punto de partida (1979) nos ubica en la dirección de un centro de documentación y el diseño e implementación de proyectos de documentación e información en un centro autónomo de investigación en ciencias sociales en Uruguay fundado en 1975 (CIESU). En él participaban un grupo de investigadores seniors sociológos, demógrafos, politólogos, y otros especialistas en ciencias sociales que habían tenido un lugar muy destacado en la Universidad de la República hasta su intervención por el gobierno autoritario (1974); existe consenso en considerarlos una parte fundamental de la "intelligentsia" uruguaya en ciencias sociales que pudo permanecer en el país. Entre ellos -aproximadamente unos 15 investigadores permanentes - destacamos al fundador del CIESU y uno de los principales sociólogos de América Latina, Carlos Filgueira; él orientó y apoyó al Centro de Documentación en la inserción y participación como nodo nacional en la Red IPALCA y en estrechar las relaciones con CLADES de CEPAL, siendo distribuidores informales de sus

7 A manera de ejemplo: Primer Taller de la Red de Información y Comunicación de CLACSO, Buenos Aires, 24-28 de abril de 1989 y el Taller de la Red de Información y Comunicación de CLACso, Santiago de Chile, 24-29 de noviembre de 1991 con motivo de la Xvi Asamblea General de CLACSO. 
"paquetes" de Formatos CEPAL en los primeros años de su difusión, hasta que asumió esta responsabilidad el CONICYT. Eran tiempos de la consolidación democrática, primeros períodos de gobierno después de la dictadura (1985-1989), y creemos que por ser un lapso de reinicio y reconstrucción se retrasaron algunos procesos y de allí que los centros continuaran — como se señaló al principio de esta presentación - cumpliendo determinadas funciones y servicios hasta el fortalecimiento universitario, después del fin de la intervención de la Universidad a mediados de los 80 .

En el lapso de este abordaje, diferenciamos las primeras etapas, 1979-1985, durante el cual el trabajo "puertas adentro" alentó una interdisciplinariedad que se tradujo poco después en proyectos de información y documentación, donde convivieron bibliotecólogos/documentalistas con sociólogos, urbanistas, politólogos e informáticos en una interacción enriquecedora. La vida cotidiana de la academia en centros pequeños nos lleva a invocar las investigaciones de esos años en el Reino Unido (Proyectos INFROSS e INISS) como las contemporáneas sobre la información para la vida cotidiana de los teóricos de los países escandinavos. Pero no es el motivo ni la extensión de esta presentación ahondar en estas consideraciones. A la vez, fue un quehacer - a pesar de las dificultadesno encapsulado, sino por el contrario se trató de crear nexos con las unidades de información de los otros centros, los conocidos como "cinco centros de CLACSO" a los cuales ya se hizo referencia, y nos conectábamos con otras ONG de la región.

A los pocos años, una segunda etapa que podemos ubicar entre mediados de los 80 y 90, las nuevas condiciones del país permitieron consolidar ese proceso "puertas afuera". Entre los ejemplos es representativo el Taller de presentación de resultados y evaluación del Proyecto SINUR apoyado por el IDRC (Sabelli, 1992), donde generadores de conocimiento, especialistas, usuarios, profesionales de la información, decisores y organismos financiadores intercambiaron y discutieron. En suma, un “dominio" específico, centrado en la cuestión urbana, mostraba sus paradigmas, conceptos, lenguajes en torno a problemática documental.

También se intentó coordinar una Red de las unidades de información de los Centros miembros de CLACSO del Uruguay, creada en 1988 y denominada REDICSU, que llegó a reunir las publicaciones de los centros en una base de datos bibliográfica.

Por último, cerrando una etapa y abriendo otra, queremos mencionar cómo el Centro de Documentación inició una línea de investigación en Uruguay, continuada hasta la actualidad en la Universidad de la República, en Estudios de Usuarios (Pérez Giffoni y Sabelli, 2010) a partir del Proyecto Usuarios en Ciencias Sociales de la Red IDIN (1992-1993), coordinado por CLACSO con el apoyo del CIID/IDRC. 
Así pues, de un modo u otro, quisimos mostrar algunas experiencias desde un centro, pero seguramente hay múltiples vivencias, iniciativas y desafíos en la memoria de cada unidad de información en ciencias sociales de aquel período que no se han compartido. Sabemos que existieron y quisiéramos recuperarlas entre todos. Invitamos a investigar a los jóvenes a la luz de los tiempos actuales.

\section{LOS PUENTES CONSTRUIDOS EN TIEMPOS DIFÍCILES DESDE LA MIRADA DE UNA ACTUALIDAD DESAFIANTE PARA LA DISCIPLINA Y LA PROFESIÓN}

Si analizamos estas experiencias compartidas vemos la necesidad de investigar sobre ellas, como forma de debatirlas y contribuir a la reflexión sobre la teoría y la praxis de las disciplinas de la información en unidades de información del área de las ciencias sociales. Deberían verse y discutirse desde distintos paradigmas; es deseable repensar las distintas etapas entre interlocutores que brinden miradas diversas.

El intercambio entre pares como comunidades de práctica no es frecuente, pero resulta imprescindible para crear una masa crítica en este sector del colectivo profesional y académico. Muchas veces, y quizás demasiado, nos hemos centrado en la formación y aplicación de nuevas tecnologías de información y comunicación (de las nuevas tecnologías de información y comunicación de los años 80 a las TIC desde mediados de los 90), es preciso preguntarnos y discutir en base a los ¿para qué?, ¿por qué? y ¿cómo nos ven "los otros"? para cuestionarnos las mejores formas de relacionarnos con el usuario-creador y en definitiva, la mejor forma de crear puentes de mediación con los recursos de información y también puentes entre las viejas y nuevas generaciones de mediadores y usuarios-creadores especialistas en ciencias sociales.

\section{REFERENCIAS BIBLIOGRÁFICAS}

Barreiro Díaz, A. (2000). A contrução social das ciencias sociaies na periferia: economía e sociología no Uruguai, 1970-1990. Tesis de doctorado en Política Científica y Tecnológica presentada en el Instituto de Geociencias de la Universidad Estadual de Campinas. Campinas, São Paulo, noviembre 2000.

Brunner, J.J. y Barrios, A. (1987). Inquisición, mercado y filantropia: ciencias sociales y autoritarismo en Argentina, Brasil, Chile y Uruguay, Santiago, Chile: FLACSO. 
IPALCA (1987). IPALCA. Documento 1987. Seminario: Información sobre población en América Latina y el Caribe: el diseño de una estrategia para la próxima década, Santiago de Chile, julio de 1987, disponible en: <http://www.un.org/popin/regional/latam/argentina/pro lap/docume87.htm>, acceso el 9 de abril 2012.

IPALCA (1993). Seminario Red IPALCA: "Aprovechando las nueva tecnología", Santiago, Chile, 16-19 de noviembre de 1993, disponible en: <http://www.un.org/popin/regional/latam/argentina/prolap /semina93.htm>, acceso el 9 de abril 2012.

NU. CEPAL (1990-1997). Serie Información y Desarrollo. N¹-8.

Paris de Oddone, B. y Alfaro, M. (1987). Las ciencias sociales en Uruguay, La Lupa. Brecha, 30 abril y 8 de mayo de 1987.

Pérez Giffoni, M.C. y Sabelli, M.(2010). Los estudios de usuarios de información: construcción de una línea de investigación y docencia en el Uruguay, Montevideo, Escuela Universitaria de Bibliotecología y Ciencias Afines, Universidad de la República

Prates, S. (1987). Los centros autónomos en ciencias sociales en el Uruguay: trayectoria y perspectivas, Montevideo, CIESU. Cuadernos del CIESU; $n^{\circ} 55$.

Sabelli, M., comp.(1992). Investigación y documentación en la cuestión urbana. Montevideo: CIESU/SINUR.

Sabelli, M. (2010) "Library and information sciences in the information disciplines environment: towards integrative models of disciplines, professional community and information and communication public policies." en Information Research, v.15, núm. 4, paper colis720, disponible en <http://InformationR.net/ir/15-4/colis720. html> el 9 de abril 2012.

Sabelli, M. y Chiacchio, A.M. (1980). Anteproyecto SICLACSO: Sistema de Información del Consejo Latinoamericano de Ciencias Sociales. Montevideo, CIESU. 